\title{
Visco-acoustic transmission waveform inversion of velocity structure in space-frequency domain*
}

\author{
Guihua Long ${ }^{1,2, \uparrow}$ Xiaofan $\mathrm{Li}^{1} \quad$ Meigen Zhang $^{1}$ and Tong Zhu ${ }^{1,2}$ \\ ${ }^{1}$ Institute of Geology and Geophysics, Chinese Academy of Sciences, Beijing 100029, China \\ ${ }^{2}$ Graduate University of Chinese Academy of Sciences, Beijing 100049, China
}

\begin{abstract}
According to the least square criterion of minimizing the misfit between modeled and observed data, this paper provides a preconditioned gradient method to invert the visco-acoustic velocity structure on the basis of using sparse matrix LU factorization technique to directly solve the visco-acoustic wave forward problem in space-frequency domain. Numerical results obtained in an inclusion model inversion and a layered homogeneous model inversion demonstrate that different scale media have their own frequency responses, and the strategy of using low-frequency inverted result as the starting model in the high-frequency inversion can greatly reduce the non-uniqueness of their solutions. It can also be observed in the experiments that the fast convergence of the algorithm can be achieved by using diagonal elements of Hessian matrix as the preconditioned operator, which fully incorporates the advantage of quadratic convergence of Gauss-Newton method.
\end{abstract}

Key words: visco-acoustic; waveform inversion; $L U$ factorization; preconditioned operator CLC number: P315.3 Document code: A

\section{Introduction}

Surface seismic data and logging data are often used to investigate the Earth's inner structure or physical properties by geophysical inversion (Yang, 1997), which in mathematics can be expressed as a process of inverting parameters through solving partial differential equations with already known solutions. This process can also be understood as a wave equation constrained nonlinear least-square problem in optimization (Akcelik, 2002).

There are many methods to handle least-square problem in mathematics, but there were few examples applying them to geophysical inversion before 1984 . The main reason may attribute to difficulties of obtaining the sensitivity matrix of the objective function with respect to model parameters at that time due to computational power. There were also other reasons, for example, theory incompleteness and shortage of effective numerical methods, which should be considered in the inversion. In order to overcome these difficulties, many researchers have done a lot in the past decades. The

\footnotetext{
* Received 12 June 2008; accepted in revised form 25 September 2008; published 10 February 2009.

* Corresponding author. e-mail: longgh@mail.iggcas.ac.cn
}

most representative one is Tarantola (1984) who recognized the gradient of the misfit function with respect to model parameters can be computed by 'backpropagating' the wave field residuals and correlating the results with forward-propagated wave fields. For there is no need to compute the sensitivity matrix, Kolb et al (1986), Gauthier et al (1986) and Mora (1987) successfully applied his method (Tarantola, 1984, 1987) to the time domain wave equation inversion. The same success was also obtained by Shin (1988), Pratt and Worthington (1990), Pratt (1990b), Geller and Hara (1993) and Song et al (1995) in the frequency domain, and several of them have even applied it to the real logging data. When comparing the results inverted by this method with those of travel-time inversion, superior resolution can be found in frequency domain waveform inversion (FWI) (Williamson, 1991; Williamson and Worthington, 1993). By using the reciprocity principle of shots and receivers, Pratt et al (1998) deepened and broadened Tarantola's method (1984) in a way of deriving efficient algorithms for gradient computation. He also studied the way of accurately inverting Hessian matrix, on which Gauss-Newton and full Newton methods are based to perform the frequency domain velocity inversion in heterogeneous media. The progresses achieved by Pratt and 
Worthington (1990), Pratt (1990a, b), Pratt et al (1998) lay a solid foundation for the successors to carry on the waveform inversion study. The following methods to get gradient and Hessian matrix in seismic waveform inversion are almost the same as Pratt's except those computed by perturbation analysis, sensitivity matrix computation or adjoint methods. This can be seen in Zhang's (2003) and $\mathrm{Xu}$ and Wang's (2004) papers, which are mainly based on finite element method and sensitivity matrix computation to invert the parameters in structured geological model. In these papers, they also analyzed the anti-noise capacity of the Leverberg-Marquard method (Marfurt, 1984) they used to solve the least-square problem. Operto et al (2007) pushed the FWI methods to a new stage. Based on optimal frequency selection methods by Sirgue and Pratt (2004) and Brenders and Pratt (2007) in migration and inversion, he concentrated on the study of frequency domain inversion of wide amplitude data which is often obtained from ocean bottom seismometers and generalized it into 3D space.

Different from time domain methods which use all frequencies, frequency domain methods use only few frequencies to invert the accurate model. The disadvantage to use all the frequencies in time domain methods lies in increasing the non-uniqueness of solutions in inversion, which makes iterative methods (e.g., conjugate gradient methods and steepest descend methods) more vulnerable to be trapped in a local minimum. But, this phenomenon can be alleviated if we use the results inverted from low frequency data as the starting model of higher frequency inversion because low frequency data which corresponds to long wavelength can resolve only the macrostructures in the media. By virtue of this merit, this paper has successfully used FWI method to do inversions in an inclusion media and a layered homogeneous media, which proves that it can be used as an effective method to assess the inner structure of the unknown media when there is no a priori information provided.

\section{Theory}

\subsection{Solving acoustic wave equation in the space- frequency domain}

The two-dimensional acoustic wave equation in the space-frequency domain can be obtained by doing Fourier transform to the time domain acoustic wave equation. The resultant system can be written as

$$
\frac{\omega^{2}}{c^{2}(x, z)} \boldsymbol{p}(x, z, \omega)+\Delta p(x, z, \omega)=\boldsymbol{s}(x, z, \omega),
$$

where $\omega$ is the angle frequency, $c(x, z)$ the acoustic velocity, $\boldsymbol{p}(x, z, \omega)$ the pressure field and $\boldsymbol{s}(x, z, \omega)$ the source.

From analysis of the visco-acoustic wave equation conducted by Niu and Sun (2002), we know that one advantage of handling forward problems of seismic wave propagation in the space-frequency domain is that one can easily consider into attenuation in wave equation by simply changing the real velocity into its complex form. According to Toksőz and Johnston (1981), it's easy to find out that the imaginary part of the complex velocity acts as an attenuation factor in numerical modeling when we use monochromatic plane wave to analyze the visco-acoustic wave equation. Assuming the quality factor is independent of frequency, the complex velocity can be written as a function of real velocity and quality factor (Ravaut et al, 2004)

$$
\frac{1}{\widetilde{c}}=\frac{1}{c}\left[1+\frac{i}{2 Q} \operatorname{sign}(\omega)\right]
$$

where $\widetilde{c}$ is the complex velocity and 'sign' is a sign function.

Substituting the real velocity in equation (1) with the complex velocity in equation (2) in the visco-acoustic wave equation yields the general form of Helmholtz equation

$$
\left[\Delta+(1-\alpha i) k^{2}(x, z, \omega)\right] \boldsymbol{p}(x, z, \omega)=\boldsymbol{s}(x, z, \omega),
$$

where $\alpha$ is an attenuation coefficient which depends on $Q, k$ is the wavenumber which equals to $\omega / c$. When discretized using a finite difference method (FDM), the above Helmholtz equation can be written in a matrix-vector multiplication form

$$
A p=s,
$$

where the complex-valued impedance matrix $\boldsymbol{A}$ depends on not only the frequency but also media properties, the 2D pressure $\boldsymbol{p}$ and source $\boldsymbol{s}$ fields at one frequency $\omega$ are stored as vectors of dimension $n x \times n z$ where $n x$ and $n z$ are dimensions of the regular FD grid with equal-spaced interval.

In the past three decades attempts to iteratively solve Helmholtz equation have been made by many authors. The paper of Bayliss et al (1983) can be considered as the first publication which shows efficient implementation of an iterative method (i.e., conjugate gradients) on the Helmholtz equation. The follow-up paper 
by Gozani et al (1984) includes multigrid as a preconditioner in the conjugate gradient algorithm. Because these methods are not of highest efficiency for high wavenumber (or frequency) problems, many contributions have been made since then. The well-designed iterative methods using optimization techniques can be found to have greater efficiency than the traditional direct solver to solve Helmholtz equation, especially in three-dimensional space. It seems iterative methods are more appealing, but when we do acoustic waveform inversion which needs abundant forward computations with multiple sources, the iterative methods still remain low efficient, because the nature of iterative methods confines themselves in the dilemma that they can not fully use the structures and characteristics of the impedance matrix. At this point, direct methods, which mainly consist of LU factorization (Press et al, 1992; Pratt, 1990b; Golub and Van Loan, 1996) and QR factorization (Golub and Van Loan, 1996), seem much better than iterative methods. Because they can fully explore the impedance matrix factorization information and different right-hand terms (sources), there is no need to refactorize the impedance matrix at one selected frequency when we shift from one source to another. This greatly reduces the computation time. In fact, most of time is consumed by matrix factorization. Besides, we also employ the bandwidth of the impedance matrix with low order and low dispersion finite difference. By using re-ordering strategy, we only need to store the non-zero elements and their index information, which makes it possible to save huge storages. Combining with the factorization algorithms of sparse matrix, the cost of time and memory will be reduced to a great extent.

\subsection{Acoustic waveform inv ersion in space-frequency domain}

Following Tarantola (1984), we formulate the inverse problem as a least-square optimization with $L_{2}$ norm of the data residuals

$$
\min E(\boldsymbol{c})=\frac{1}{2}\left\|\boldsymbol{p}(c)-\boldsymbol{p}_{\mathrm{obs}}\right\|_{2}^{2},
$$

where $\boldsymbol{p}(c)$ is the pressure wavefield estimated from the current model $c$ and $\boldsymbol{p}_{\mathrm{obs}}$ the observed pressure wavefield.

Assume that we have gotten the solution $c_{k}$ at step $k$ with an iterative method, doing Taylor expansion to the misfit function at this reference point will result in transforming the space-frequency domain wave equation inversion into a nonlinear quadratic optimization prob- lem

$$
\min m_{k}(\boldsymbol{d})=E_{k}+\nabla E_{k}^{\mathrm{T}} \boldsymbol{d}+\frac{1}{2} \boldsymbol{d}^{\mathrm{T}} \nabla^{2} E_{k} \boldsymbol{d},
$$

where $\boldsymbol{d}$ is the increment of velocity model, $\nabla E_{k}$ the gradient of the misfit function and $\nabla^{2} E_{k}$ the Hessian matrix.

The key to solve equation (6) depends on Jacobian matrix calculation, because how well the Jacobian matrix computes determines the accuracy of the gradient, the Hessian and the regularization factor in the inversion. Taking the partial derivative of both sides of equation (4) with respect to the $i$ th parameter $c_{i}$, we can get the derivative wavefield from the following equation

$$
A \frac{\partial \boldsymbol{p}}{\partial c_{i}}=\overline{\boldsymbol{f}}_{i}
$$

where $\overline{\boldsymbol{f}}_{i}$ denotes the virtual source that can be represented as

$$
\overline{\boldsymbol{f}}_{i}=-\frac{\partial A}{\partial c_{i}} \boldsymbol{p}
$$

By analogy with equation (4), the partial derivatives in equation (7) are the solution of a newly formed forward-modeling problem driven by the virtual sources at the location of the $i$ th parameter. Arranging all derivatives by model parameters, Jacobian matrix can be represented simultaneously by the matrix equation

$$
\boldsymbol{J}=\left[\frac{\partial \boldsymbol{p}}{\partial c_{1}}, \frac{\partial \boldsymbol{p}}{\partial c_{2}}, \cdots, \frac{\partial \boldsymbol{p}}{\partial c_{N}}\right]=\boldsymbol{A}^{-1} \boldsymbol{F},
$$

where $N$ is the total number of model parameters, $\boldsymbol{F}=\left[\overline{\boldsymbol{f}_{1}}, \overline{\boldsymbol{f}_{2}}, \cdots, \overline{\boldsymbol{f}_{N}}\right]$ the virtual source term. Note that the inverse of matrix $\boldsymbol{A}$ probably does not exist, to write here is just for simplicity.

The gradient can be obtained by taking partial derivative to the misfit function in equation (5) with respect to the model parameters

$$
\nabla E=\mathfrak{R e}\left\{\boldsymbol{J}^{\mathrm{T}} \delta \boldsymbol{p}^{*}\right\}
$$

where $\delta \boldsymbol{p}$ is the data residual between model response and the observed data at the receiver locations, the asterisk indicates complex conjugate, and ' $R e$ ' denotes the real part of a complex number.

Substituting the Jacobian matrix in equation (9) into equation (11), we obtain

$$
\nabla E=\Re \mathrm{R}\left\{\boldsymbol{F}^{\mathrm{T}}\left[\boldsymbol{A}^{-1}\right]^{\mathrm{T}} \boldsymbol{\delta} \boldsymbol{p}^{*}\right\} .
$$

Let $v$ equal to the multiplication of the last two terms in above equation 


$$
\boldsymbol{v}=\left[\boldsymbol{A}^{-1}\right]^{\mathrm{T}} \delta \boldsymbol{p}^{*},
$$

it is clear to see that $v$ is the 'backpropagated' wavefield of the residual data. When considering into equation (11) the virtual source term $\boldsymbol{F}$ arranged by equation (8), the gradient of the misfit function can be written as

$$
(\nabla E)_{i}=\Re\left(\boldsymbol{p}^{T}\left[\frac{\partial \boldsymbol{A}^{\mathrm{T}}}{\partial c_{i}}\right] \boldsymbol{v}\right\},
$$

where we notice that the gradient can be presented as some kind of inner product of forward wavefield with data residual 'backpropagated' wavefield. However, in the time domain, this can be represented as the zero-lag cross-correction of the forward wavefield and the 'backpropagated' wavefield (Mora, 1987).

In order to calculate the second-order partial derivatives of the misfit function with respect to the model parameters, we need to do another differentiation in equation (7) with respect to $c_{j}$. The derived formula is

$$
\boldsymbol{A} \frac{\partial^{2} \boldsymbol{p}}{\partial c_{i} \partial c_{j}}=-\frac{\partial \boldsymbol{A}}{\partial c_{j}} \frac{\partial \boldsymbol{p}}{\partial c_{i}}-\frac{\partial^{2} \boldsymbol{A}}{\partial c_{i} \partial c_{j}} \boldsymbol{p}-\frac{\partial \boldsymbol{A}}{\partial c_{i}} \frac{\partial \boldsymbol{p}}{\partial c_{j}} .
$$

Just like the way to deal with virtual source term in equation (7), we can treat the right-hand term in the above equation as the secondary virtual source. By sorting the second-order partial derivative wavefield, we can get the Hessian matrix

$$
\begin{aligned}
\boldsymbol{H}_{i j} & =\frac{\partial^{2} E}{\partial c_{i} \partial c_{j}}=\Re \mathrm{Re}\left\{\boldsymbol{J}^{\mathrm{T}} \boldsymbol{J}^{*}\right\} \\
& +\Re\left[\left(\frac{\partial \boldsymbol{J}^{\mathrm{T}}}{\partial c_{1}} \delta \boldsymbol{p}^{*}\right)\left(\frac{\partial \boldsymbol{J}^{\mathrm{T}}}{\partial c_{2}} \delta \boldsymbol{p}^{*}\right) \ldots\left(\frac{\partial \boldsymbol{J}^{\mathrm{T}}}{\partial c_{N}} \delta \boldsymbol{p}^{*}\right)\right]
\end{aligned}
$$

The biggest difficulty to invert the Hessian matrix is that calculating the second term in equation (15), including secondary information which is helpful for model reconstruction, is almost impossible even by up-to-date supercomputers. Due to the uniqueness of this problem, it is also not reasonable to use the secant approximation of the whole $\nabla^{2} E_{k}$. In order to find a way out, we have to generally simplify the computation by discarding the second term in the right-hand of the equation (15). It is natural to do this because in weak inhomogeneous media this term is always trivial when comparing with the first term. Therefore, the Hessian matrix in equation (15) can be simplified as

$$
\boldsymbol{H}_{a}=\frac{\partial^{2} E}{\partial c_{i} \partial c_{j}}=\Re \operatorname{Re}\left\{\boldsymbol{J}^{\mathrm{T}} \boldsymbol{J}^{*}\right\} .
$$

The method to solve the nonlinear quadratic opti- mization problem using the above approximate Hessian is Gauss-Newton method, which has been fully discussed by Pratt et al (1998) in his paper. Although Pratt et al (1998) has done good job in Hessian computation, it is still unwise to exploit the whole Hessian matrix in practical inversion especially in large-scale inhomogeneous media even if the full Hessian matrix is strictly diagonally dominant and the off-diagonal elements which represent multiple scattering can accelerate the convergence of the optimization process. Because in weak inhomogeneous media the off-diagonal elements of the Hessian matrix are usually small and the computation of them is intensive, so there is no need to compute the full Hessian matrix but only the diagonal elements in the inversion. By analyzing the process of deriving Hessian matrix, we find the elements of the Hessian matrix

$$
\boldsymbol{H}_{i j}=\frac{\partial^{2} E}{\partial c_{i} \partial c_{j}}=\Re\left(\boldsymbol{p}^{\mathrm{T}} \frac{\partial \boldsymbol{A}^{\mathrm{T}}}{\partial c_{i}}\left(\boldsymbol{A}^{-1}\right)^{\mathrm{T}}\left(\boldsymbol{A}^{-1}\right)^{*} \frac{\partial \boldsymbol{A}^{*}}{\partial c_{j}} \boldsymbol{p}^{*}\right\}
$$

can be represented as the cross-correction of partial derivative wavefields. If we let $i=j$ in the above formula, we can get the diagonal elements of the Hessian. Using the diagonal elements to approximate the full Hessian matrix in the inversion is reasonable because it is easy to guarantee that the data residuals between the calculated wavefield and the observed wavefield are small under weak inhomogeneous media condition.

Using the diagonal Hessian to represent the real Hessian, nonlinear quadratic optimization of the space-frequency domain wave equation inversion in equation (6) can be turn into

$$
\min m_{k}(\boldsymbol{d}) \approx E_{k}+\nabla E_{k}^{\mathrm{T}} \boldsymbol{d}+\frac{1}{2} \boldsymbol{d}^{\mathrm{T}}\left\{\operatorname{diag} \boldsymbol{H}_{a}+\beta \boldsymbol{I}\right\} \boldsymbol{d},
$$

where $\beta$ is the regularization factor which is used to guarantee the positive definiteness of the second-order term. According to KKT condition, the above optimization problem can be solved and we finally get the iterative increment

$$
\boldsymbol{d}^{(k)}=-\alpha\left(\operatorname{diag} \boldsymbol{H}_{a}+\beta \boldsymbol{I}\right)^{-1} \nabla E_{k},
$$

where $\alpha$ is the step length which can be calculated by line-search techniques. By analogy to the pure gradient methods, using diagonal Hessian as a preconditioner (Shin et al, 2001; Operto et al, 2007) can control well the amplitudes of the perturbation and absorb the quadratic convergence of Gauss-Newton method. It also makes the inversion method proposed in the paper have 
fast convergence.

\section{Numerical tests}

In order to test the robustness of the above proposed FWI method in complex media, two models are designed. One is an inclusion model with three inhomogeneous bodies implanted in a homogeneous background medium, and the other is a layered homogeneous model with uneven interfaces. The inclusion velocity model used in this study is shown in Figure 1 (True). The velocities of the three inhomogeneous bodies varying from up to down are $4200 \mathrm{~m} / \mathrm{s}, 4500 \mathrm{~m} / \mathrm{s}$ and 3800 $\mathrm{m} / \mathrm{s}$, and the background velocity is $4000 \mathrm{~m} / \mathrm{s}$. To more realistically model the Earth model, quality factor is also considered in the modeling. Here, we assume the media attenuation is weak and the quality factor equals to 10 000 . The whole model was divided into $201 \times 201$ grids with an interval of $25 \mathrm{~m}$. Shots were deployed with a 50 $\mathrm{m}$ spacing along the top and left edges of the model.
Shots on the top and left edges were recorded by 99 receivers located on the bottom and right edges respectively. The distance between shots or receivers and the edges of the model is $100 \mathrm{~m}$.

In forward modeling, 4096 frequencies ranging from 0.0 to $50 \mathrm{~Hz}$ were used with an interval of 0.0122 Hz. For the inversion run, only 8 frequencies $(2.0,4.0$, 5.0, 7.0, 10.0, 13.0, 16.0 and $20.0 \mathrm{~Hz}$ ) were inverted sequentially in the inclusion model. It started at $2.0 \mathrm{~Hz}$ with the initial model equal to the background velocity. After tens of iterations, we got the inverted result as shown in Figure 1a. Then using this inverted result as the initial model for the next frequency and going on until all frequencies finished, we, at last, got the final inverted velocity model (seen in Figure $1 \mathrm{~h}$ ).

As we can see in Figure 1, all three inhomogeneous bodies can be recognized clearly except the result inverted from $2.0 \mathrm{~Hz}$ that only distinguishes the big one. The resolution is getting better with frequency increasing.
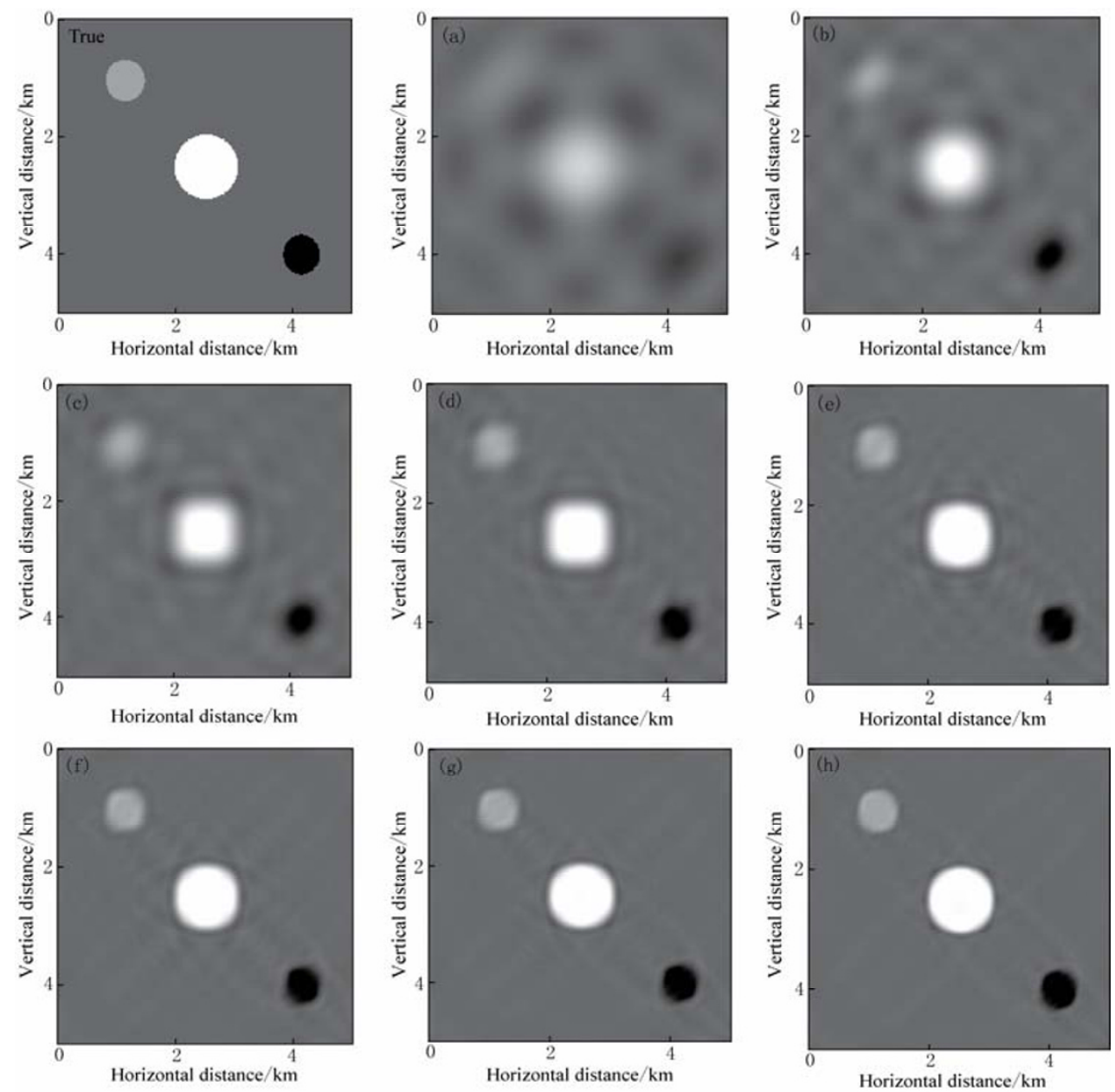

Figure 1 True velocity model and inverted velocity structure of the inclusion model. True: the target model, (a) (h): inverted velocity structure at individual frequency of 2.0, 4.0, 5.0, 7.0, 10.0, 13.0, 16.0 and $20.0 \mathrm{~Hz}$, respectively. 
It should be not hard to explain why this happens. Through further study and observation, you will find that the resolution have a close relation with frequency and wavelength. For those wavefields with low frequency, they are more sensitive to the macrostructures than the small ones. Such a merit greatly reduces the searching space of velocity model in inversion, which, from an optimization point, can substantially improve the non-uniqueness of the nonlinear quadratic misfit function. As shown in Figure 2, comparisons between the velocity models inverted from different frequencies and the true velocity model demonstrate that as for using local optimization methods and there is no a priori information about the initial model, it is reasonable to start the inversion from low frequency. This kind of strategy is like the multiscale method (Bunks et al, 1995; Feng, 2006) in the time domain, which first operates on the coarse grids to invert a coarse model and then interpolates them on finer grids to alleviate the non-uniqueness of the solution in the iterative inversion.

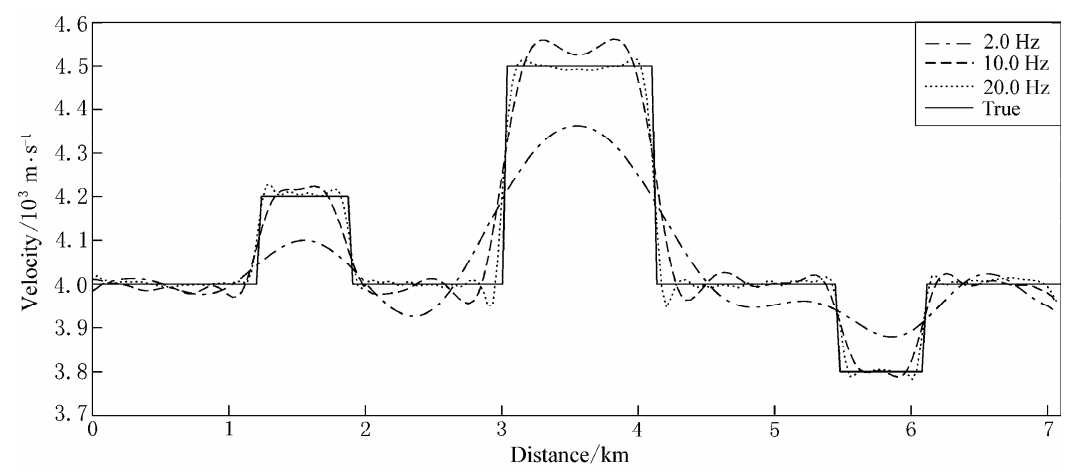

Figure 2 Comparison between inverted velocity structures computed at different frequencies and the true velocity model.

In the layered homogeneous model, the shots and receivers are configured as the same as in the inclusion model. As we can see in Figure 3 (True), the real velocities in the layered media changing from top to bottom are $3800 \mathrm{~m} / \mathrm{s}, 3500 \mathrm{~m} / \mathrm{s}, 4000 \mathrm{~m} / \mathrm{s}, 4200 \mathrm{~m} / \mathrm{s}$ and 4300 $\mathrm{m} / \mathrm{s}$. There is a near-surface low velocity layer below the overlaying media with a thickness of about $450 \mathrm{~m}$. The layer is $500-600 \mathrm{~m}$ deep on the left edge and extending to about $1000 \mathrm{~m}$ deep on the right edge. The velocities in the following layers increase with depth. The interfaces between any two adjacent layers change slightly as that in the low velocity layer. From the inverted results in Figures 3b-3h, we can see that different frequencies have different resolutions. Low frequencies can resolve the macrostructure of the media, and high frequencies refine the results inverted by low frequencies. The comparisons between the inverted models from different frequencies also show that although low frequencies can only provide a coarse structure, they can be treated as a good starting model for the high frequency inversion. To do this can greatly alleviate the non-uniqueness of the nonlinear iterative optimization inversion.

By analyzing the velocities in Figure 4, we can also find that even with a frequency of $10 \mathrm{~Hz}$ the inverted result achieves a good approximation to the true model except some trivial difference near the surface or on the interfaces. The following high frequencies contribute fewer to the final result. One reason why this happens may attribute to the regularization method, which in the paper only considers adding a trivial quantity that makes Hessian matrix positively definite to guarantee a minimum in the optimization. The best way many authors suggested solving this problem is the total variation method which is adaptive to sharp parameter changing. This is not to say that we should put all blames on regularization. To invert accurate model, we still need to find the missing seismic trace information and improve the used method in inversion.

By analyzing the resolution of every frequency, it's easy for us to explain why $10 \mathrm{~Hz}$ is enough to invert an accurate result. The frequencies greater than $10 \mathrm{~Hz}$ seem redundant because they contribute little to the final result but only do a refining (see Figure 4). This means that if we want to get the results with the same accuracy, we can reduce the frequencies we processed in the tests. In fact, the optimal frequency has a close relation with offset. The relation has been verified by Sirgue in 2004 when he analyzed the migration and inversion in space-frequency domain. In his paper, he also gave the optimal frequency selection method. 

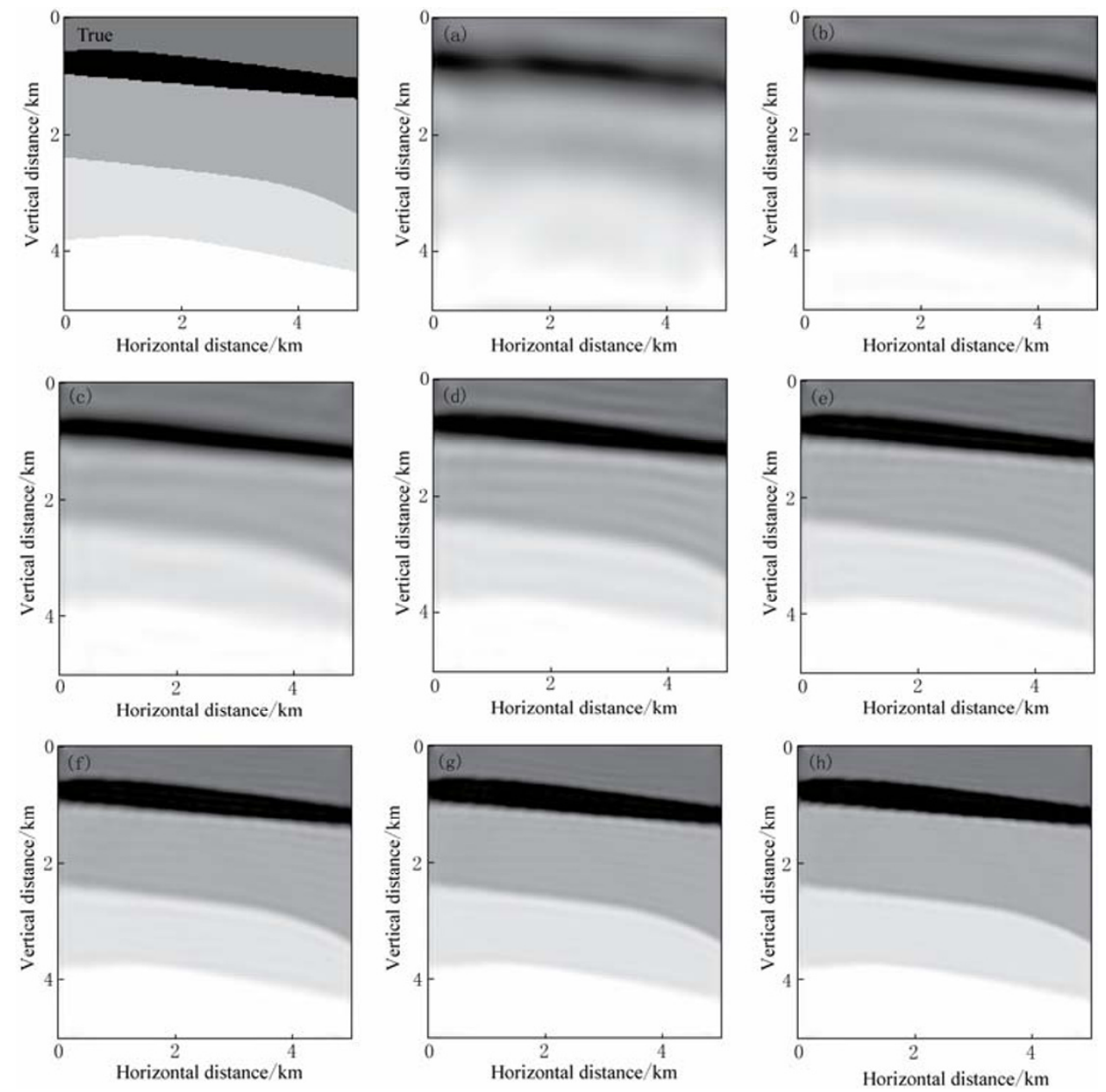

Figure 3 True velocity model and inverted velocity structures of layered model. True: the target model, (a)-(h): inverted velocity structures at individual frequency of 2.0, 4.0, 5.0, 7.0, 10.0, 13.0, 16.0 and $20.0 \mathrm{~Hz}$, respectively.

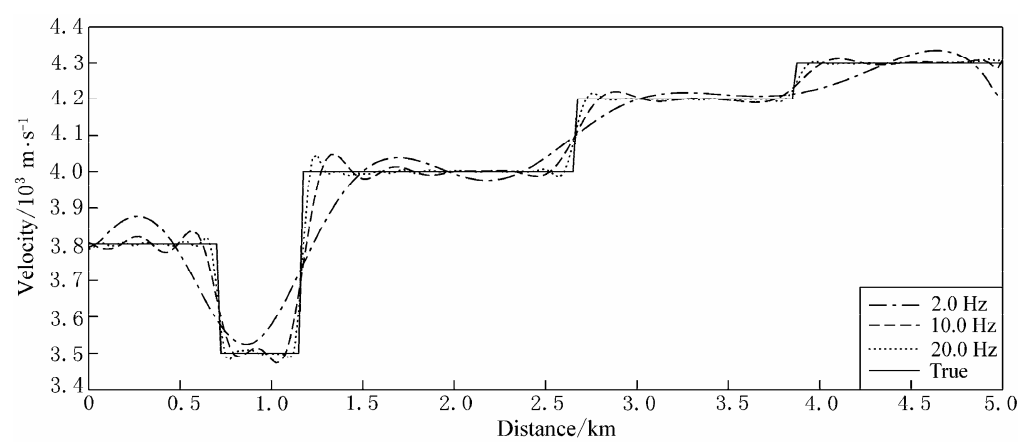

Figure 4 Comparison between inverted velocity structures computed at different frequencies and the true velocity model.

\section{Discussion and conclusions}

The preconditioned steepest descent method based on the diagonal Hessian has been derived in this paper to solve the least-square inversion problems in the space-frequency domain. Tests using this method in lo- cal inhomogeneous media and layered homogeneous model demonstrate that only several frequencies are needed to invert the accurate velocity model. The tests also show that low frequency data are sensitive to macrostructure and using the result inverted from low frequency data as the starting model of high frequency in- 
version can drastically lessen the non-uniqueness of the badly-posed inversion problem.

In spite of deploying the space-frequency domain method makes it possible to obtain good results in local inhomogeneous media and layered homogeneous media, there is no theory to prove that the hypothesis of approximating the Hessian matrix using its diagonal elements is reasonable. The models we tested are weak inhomogeneous, whether the method is suitable for strong inhomogeneous media is still under question. Although using the diagonal Hessian as a preconditioner can accelerate the convergence rate, the discarded off-diagonal elements may be indispensable for inverting the accurate model. At this point, studies to solve the dense matrix-based linear equations introduced by the off-diagonal Hessian appear to be more vital. In sequential inversion, we can see that different frequencies do different contribution to the final result, which means that improper frequency selection would result in data redundancy and increasing the time cost. Therefore, it is necessary to further study the frequency responses of multiscale structures and the way to choose optimal frequencies, which finally make inversion more efficient.

\section{References}

Akcelik V (2002). Multiscale Newton-Krylov Method for Inversion Acoustic Wave Propagation. Carnegie Mellon University, Pittsburgh, Pennsylvania, $6-7$.

Bayliss A, Coldstein C I and Turkel E (1983). An iterative method for Helmholtz equation. J Comput Phys 49: 433-457.

Brenders J and Pratt R G (2007). Efficient waveform tomography for lithospheric imaging: Implications for realistic, two-dimensional acquisition geometries and low-frequency data. Geophys J Int 168(1): 152-170.

Bunks C, Saleck F M, Zaleski S and Chavent G (1995). Multiscale seismic waveform inversion. Geophysics 60(5): 1 457-1 473.

Feng G F (2006). Multi-scale Trust Region Inversion Methods for Inverse Problem of Wave Equation. Harbin University of Industry, Heilongjiang, $48-60$.

Gauthier O, Virieux J and Torantola A (1986). Two-dimensional non-linear inversion of seismic waveforms: numerical results. Geophysics 51(7): 1387-1 403.

Geller R J and Hara T (1993). Two efficient algorithms for iterative linearized inversion of seismic waveform data. Geophys J Int 115(3): 699-710.

Golub G H and Van Load C F (1996). Matrix Computations. The Johns Hopkins University Press, Baltimore, 1-694.

Gozani J, Nachshon A and Turkel E (1984). Conjugate gradient coupled with multigrid for an indefinite problem. Advances in Computation Methods for PDEs 14: 425-427.

Kolb P, Collino F and Lailly P (1986). Pre-stack inversion of a 1-D medium. Proc IEEE 74(3): 498-508.
Marfurt K J (1984). Accuracy of finite-difference and finite-element modeling of the scalar and elastic wave equations. Geophysics 49(5): 533-549.

Niu B H and Sun C Y (2002). Half-space Media and Seismic Propagation. China Petroleum Industry Press, Beijing, 130-150.

Mora P R (1987). Nonlinear two-dimensional elastic inversion of multioffset seismic data. Geophysics 52(9): 1 211-1 228.

Operto S, Virieux J, Amestoy P, L'Excellent J Y, Giraud L and Ali B H (2007). 3D finite-difference frequency-domain modeling of visco-acoustic wave propagation using a massively parallel direct solver: A feasibility study. Geophysics 72(5): 195-211.

Pratt R G and Worthington M H (1990). Inverse theory applied to multi-source cross-hole tomography, Part I: Acoustic wave-equation method. Geophys Prosp 38(3): 287-310.

Pratt R G (1990a). Inverse theory applied to multi-source cross-hole tomography, Part II: Elastic wave-eqaution method. Geophys Prosp 38(3): 311-330.

Pratt R G (1990b). Frequency-domain elastic wave modeling by finite differences: A tool for crosshole seismic imaging. Geophysics 55(5): 626-632.

Pratt R G, Shin C S and Hicks G J (1998). Gauss-Newton and full Newton methods in frequency-space seismic waveform inversion. Geophy $J$ Int 133(2): 341-362.

Press W H, Teukolsky S A, Vetterling W T and Flannery B P (1992). Numerical Recipes in FORTRAN: The Art of Scientific Computing. Cambridge University Press, New York, 1-992.

Ravaut C, Operto S, Improta L, Virieux J, Herrero A and Dell'Aversana P (2004). Multiscale imaging of complex structures from multifold wide-aperture seismic data by frequency-domain full-waveform tomography: Application to a thrust belt. Geophys J Int 159(3): 1 032-1 056.

Shin C S (1988). Nonlinear Elastic Wave Inversion by Blocky Parameterization. University of Tulsa, Tulsa, 4-102.

Shin C, Yoon K, Marfurt K J, Park J, Yang D, Lim H Y, Chung S and Shin S (2001). Efficient calculation of a partial derivative wavefield using reciprocity for seismic imaging and inversion. Geophysics 66(6): 1 856-1 863.

Sirgue L and Pratt R G (2004). Efficient waveform inversion and imaging: A strategy for selecting temporal frequencies. Geophysics 69(1): 231-248

Song Z M, Williamson P R and Pratt R G (1995). Frequency-domain acoustic-wave modeling and inversion of crosshole data: Part II-Inversion method, synthetic experiments and real-data results. Geophysics $\mathbf{6 0}(3)$ : 796-809.

Tarantola A (1984). Inversion of seismic reflection data in the acoustic approximation. Geophysics 49(8): 1 259-1 266.

Tarantola A (1987). Inverse Problem Theory: Method for Data Fitting and Model Parameter Estimation. Amsterdam: Elsevier, 6-332.

Toksőz M N and Johnston D H (1981). Seismic Wave Attenuation. Society of Exploration Geophysicists, Tulsa, OK, 3-74.

Williamson P R (1991). A guide to the limits of resolution imposed by scattering in ray tomography. Geophysics 56(2): 202-207.

Williamson P R and Worthington M H. 1993. Resolution limits in ray tomography due to wave behavior: Numerical experiments. Geophysics 58(5): 727-735.

Xu Kun and Wang M Y (2004). Frequency-domain finite-element inversion of elastic-wave velocity using the geological regular-blocky-model method. Chinese J Geophys 47(4): 708-717 (in Chinese with English abstract).

Yang W C (1997). Theory and Methods of Geophysical Inversion. Geological Publishing House, Beijing, 4-14 (in Chinese).

Zhang M G, Wang M Y and Li X F (2003). Full wavefield inversion of anisotropic elastic parameters in the time domain. Chinese J Geophys 46(1): 94-100 (in Chinese with English abstract). 